\title{
PASTIS: A program for curve and distribution analyses
}

\author{
DENIS COUSINEAU and SERGE LAROCHELLE \\ University of Montreal, Montreal, Quebec, Canada
}

\begin{abstract}
Reaction time (RT) data afford different types of analyses. One type of analysis, called "curve analysis," can be used to characterize the evolution of performance at different moments over the course of learning. By contrast, distribution analysis aims at characterizing the spread of RTs at a specific moment. Techniques to deduce free parameters are described for both types of analyses, given an a priori choice of the curve or distribution one wants to fit, along with statistical tests of significance for distribution analysis: The log likelihood technique is used if the probability density function is given; otherwise, a root-mean-square-deviation minimization technique is used. A program-PASTIS-that searches for the optimal parameters of the following curves is presented: power law, exponential, and e-based exponential. PASTIS also searches for Weibull and the ex-Gaussian distributions. Some tests of the software are presented.
\end{abstract}

Curve analysis can be used to characterize reaction times (RT) collected over different sessions. The chief concern of this type of analysis is the improvement of RT; more specifically, the rate of the improvement and the performance that would be expected after an infinite amount of practice (Newell \& Rosenbloom, 1981). By contrast, distribution analysis is usually concerned with RTs obtained during one session. Distribution analysis can be used to locate outlier RTs and to test models of RT data (Ratcliff, 1978).

These two types of analyses are not mutually exclusive, but complementary. Curve analyses can describe mean RTs at different moments in time, while distribution analyses give a closer look at a specific moment. In fact, the results of curve and distribution analyses performed on a given set of data should converge in order to provide a coherent interpretation.

To perform curve and distribution analyses, one must first select likely functions to fit the data. Some candidate distributions for RTs are the log normal and the gamma, also known as the Erlagian (Ulrich \& Miller, 1994). Other distributions have also been considered (see Luce, 1986). In this paper, we focus on the ex-Gaussian and the Weibull distributions. With respect to curve analysis, we are concerned mostly with the famous power law and a possible alternative, the exponential curve.

Techniques used to compute the values of the free parameters of these various functions are discussed first.

This research was done while D.C. was working on a $\mathrm{PhD}$ in psychology at the Université de Montréal. We would like to thank Guy Lacroix for his thorough revision of this report. We would also like to thank A. Heathcote, W. E. Hockley, and R. Ulrich for their reviews of an earlier draft of this paper. A postscript version of this paper is also available at http://prelude.psy.umontreal.ca/ cousined/pastis. Correspondence should be addressed to S. Larochelle, Département de Psychologie, Université de Montréal, C. P. 6128, Succ. Centre-ville, Montréal, PQ, Canada H3C 3J7 (e-mail: cousined@psy.umontreal.ca).
Then, a program called PASTIS is presented that can perform the analyses discussed. Finally, the results of tests made to evaluate the capabilities of the program are presented.

\section{CURVE ANALYSIS}

Curve analysis of RT data is often done in studies concerned with automatization of performance (Kramer, Strayer, \& Buckley, 1990; Lassaline \& Logan, 1993; Logan, 1988). Figure 1 illustrates the evolution of performance that typically occurs over practice by showing the results of a fictional nine-session experiment. Mean RTs per session are presented along with a curve that fits them. This plot has three parameters of interest: the value of performance prior to any learning (Parameter b), the value of performance after infinite learning (Parameter a), and the rate (c) at which performance drops toward the asymptote, usually called the "learning rate."

Current theories of automatization posit that practice accounts for changes in mean RTs. However, mean RT is not the only measure of performance that can be used; one might be interested in the behavior of median RT, for example. Instead of summary measures, one might also attempt to fit raw data. It is important to note that these fits may yield very different results, since the functions considered are nonlinear. It is therefore important that researchers choose the appropriate measure of performance in view of the theory tested. In this section, we discuss mean RTs. However, PASTIS can handle raw data as well as summary measures.

\section{The Power Curve}

In a very influential paper, Newell and Rosenbloom (1981) argued that the equation that best characterizes the relation between mean RT $(M)$ and sessions of practice $(N)$ is 


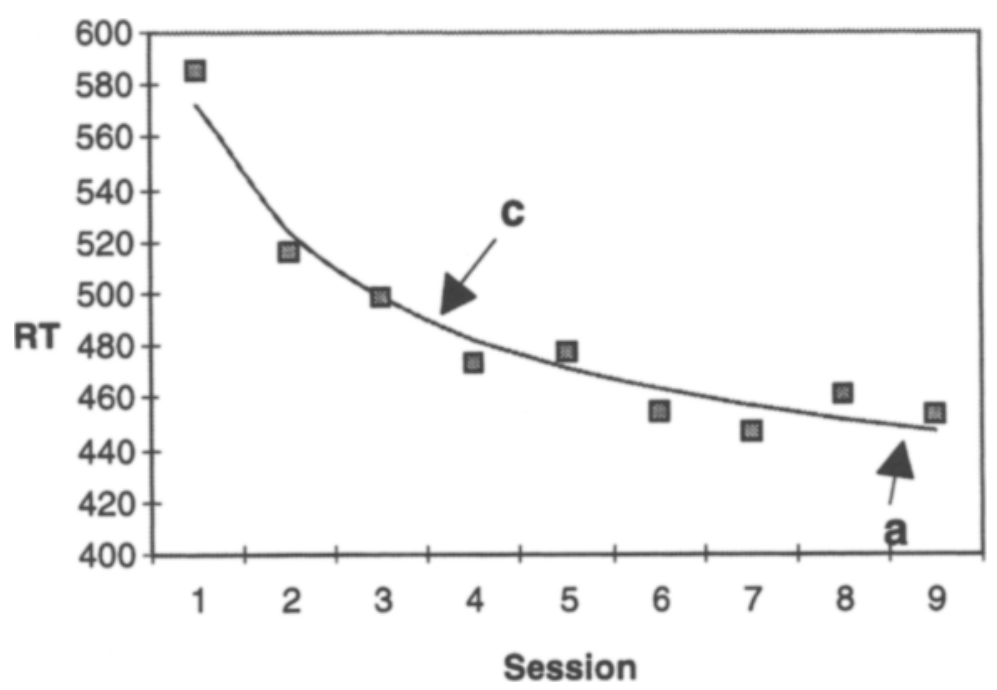

Figure 1. Simulated data and the curve that best describes the speed-up in reaction time (RT, in milliseconds).

$$
M=a+b(N+e)^{-c},
$$

where $a, b$, and $c$ represent the theoretical asymptote, the starting value, and the learning rate, and where $e$ quantifies the amount of preexperimental practice. Since most researchers assume that subjects are totally naive toward the task, they set $e$ to zero (see, however, Heathcote \& Mewhort, 1995). The most common form of the power law is thus stated as

$$
M=a+b N^{-c} .
$$

The free parameters are constrained to be greater or equal to zero, and, in the exponential curve presented later (Equation 5), the $c$ parameter must be smaller or equal to 1 since it is a rate of learning, expressed in percentage. The search for the best possible values of the free parameters is done using a minimization algorithm. The algorithm used in PASTIS is STEPIT (Chandler, 1965). ${ }^{1} \mathrm{An}$ other search algorithm, PRAXIS, was developed by Brent (1973) and implemented in C by Gegenfurtner (1992). Although we did not proceed to a systematic exploration of the capacities of both algorithms, our experience with curve analysis showed that PRAXIS converged faster on a set of estimates than did STEPIT. However, parameters obtained using STEPIT often provided a better fit of the data. For distribution analysis, the two algorithms performed equally well. We therefore decided to incorporate STEPIT into.PASTIS.

The value that PASTIS attempts to minimize is the rootmean-square-deviation (RMSD) statistic given in Equation 2, which reduces discrepancies between expected and observed means. Note, however, that there is no guarantee that the minimization algorithm will yield the smallest RMSD value. Indeed, the multidimensional space defined by the possible parameter values along with the corresponding RMSD may contain numerous local minima in which the algorithm can get trapped. The RMSD is as follows:

$$
\operatorname{RMSD}=\sqrt{1 / n \sum_{i}^{n}\left(E_{i}-O_{i}\right)} .
$$

In Equation 2, $i$ indexes the $n$ sessions, $O_{i}$ is the observed mean RT at session $i$, and $E_{i}$ is the expected mean $\mathrm{RT}$ at session $i$ given a set of parameters $a, b, c$.

One approach for testing the significance of the fit would be to linearize the relation between RTs and time of evaluation $N$ and use tests based on least square methods. Subtracting $a$ from both sides of Equation 1 yields $M-$ $a=b N^{-c}$. Computing the $\log$ arithm gives $\log (M-a)=$ $\log (b)-c \log (N)$, a linear function with a slope of $-c$ and an intercept of $\log (b)$. However, least squares methods usually require that experimental error be independent, additive, and normally distributed. But, as Sternberg (1969) pointed out, these assumptions, if valid before the transformation, are certainly destroyed after the logarithmic transformation. The Kolmogorov-Smirnov test (also called the Kolmogorov one-sample test; see Conover, 1980 ) could be used to test the normality of the residuals. However, Heathcote, Popiel and Mewhort (1991) preferred to use the nonparametric Wilcoxon sign test (see Bates $\&$ Watts, 1988, for further reading on this issue).

\section{A Special Case of the Power Law Curve Analysis: \\ Logan's Theory}

Logan's (1988) exemplar theory of automatization predicts that both the mean $(M)$ and the standard deviation $(S D)$ of RTs should decrease with practice following a power law function and that the learning rate should be the same for both measures. Equations relating mean RT $(M)$ and $S D$ are thus:

$$
M=a^{\prime}+b^{\prime} N^{-c},
$$

and

$$
S D=a^{\prime \prime}+b^{\prime \prime} N^{-c} .
$$


PASTIS can be used to simultaneously fit $M$ and $S D$ with a common $c$ parameter.

\section{The Exponential Curve}

Heathcote and Mewhort (1995) recently contested the power law of practice, suggesting that the exponential curve could provide a better characterization of the evolution of RTs. One reason for rejecting the power law is that the parameters obtained often have implausible values. Heathcote and Mewhort's survey of the literature on power law showed that the asymptote $a$, for instance, is often found to be equal to zero. Exponential fits do not show the same pathology.

The exponential function relating mean RT $(M)$ to practice session $N$ is as follows:

$$
M=a+b(1-c)^{N},
$$

where $a, b$, and $c$ have the same interpretations as in the power function. However, learning rates $c$ are on a different scale. ${ }^{2}$

Equation 5 can be linearized using a log transformation of the $M-a$ value. However, this function will be linear with respect to $N$, instead of $\log (N)$ for the power curve. The exponential Equation 5 has been used by Rescorla and Wagner (1973) as a model for associative strength in Pavlovian conditioning.

\section{DISTRIBUTION ANALYSIS}

Distribution analyses have been done in tasks such as visual search (Hockley, 1984; Ratcliff, 1978), recognition
(Ratcliff \& Murdock, 1976), letter arithmetic (Logan, 1992), and signal detection (Hohle, 1965). They have also been used to generate simulated data and to test assumptions about truncation or use of medians (Ratcliff, 1993; Ulrich \& Miller, 1994).

RT distributions can be represented in a variety of ways (Luce, 1986). The two best known are the probability density function (PDF), which gives the probability of observing a certain RT, and the cumulative function (Figure 2). Information about the central tendency and the standard deviation, as well as the skewness (seen as an asymmetry in the distribution), is best visualized using the PDF.

\section{The Ex-Gaussian Distribution}

Originally used by McGill in 1963, the ex-Gaussian distribution is a combination (convolution) of a Gaussian (normal) distribution and an exponential distribution. This distribution is known to provide a good fit for RT data in different tasks. The PDF is formulated as (Dawson, 1988; Heathcote, 1996)

$$
f(\mathrm{RT})=\frac{1}{\tau} e^{\frac{\mu}{\tau}+\frac{\sigma^{2}}{2 \tau^{2}}-\frac{\mathrm{RT}}{\tau}} \Phi\left(\frac{\mathrm{RT}-\mu-\sigma^{2} / \tau}{\sigma}\right),
$$

where $\tau$ stands for the average of the exponential component, $\mu$ and $\sigma$ are the mean and the standard deviation of the normal component, and $\Phi$ expresses the standard Gaussian integral (see Kennedy \& Gentle, 1980, for a numerical approximation). The mean of such a distribution is $\mu+\tau$, while the standard deviation is $\left(\sigma^{2}+\tau^{2}\right)^{1 / 2}$.
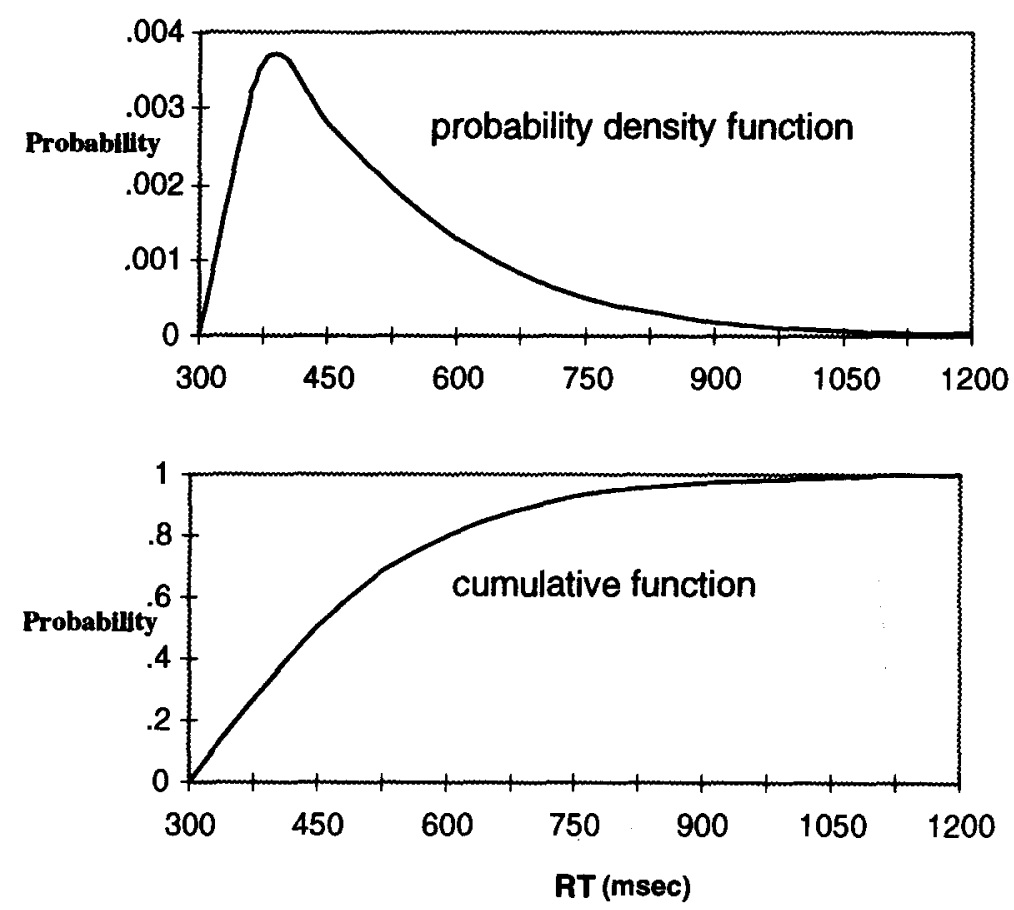

Figure 2. Two representations of a distribution. 


\section{The Weibull Distribution}

The Weibull is known to describe the distribution of minima from independent samples of identically distributed random variables. It has been used by Logan (1992) to further support his exemplarist theory of automaticity.

The cumulative function of a Weibull is given by the following formula:

$$
F(\mathrm{RT})=1-e^{-\left(\frac{\mathrm{RT}-a}{b}\right)^{c}},
$$

where $a$ and $b$ are additive and multiplicative scaling factors and $c$ is an exponential factor, sometimes called the "shape factor." The mean and the standard deviation of a Weibull are expressed by the following:

$$
\begin{aligned}
M(\mathrm{RT}) & =b \Gamma(1+1 / c)+a, \\
S D(\mathrm{RT}) & =b[\Gamma(1+2 / c)-\Gamma(1+1 / c)],
\end{aligned}
$$

where $\Gamma$ is the gamma function [if $x$ is integer, $\Gamma(x+1)$ return $x$ !].

The PDF of a Weibull is given by the following:

$$
f(\mathrm{RT})=\frac{c}{b^{c}}(\mathrm{RT}-a)^{c-1} e^{-\left(\frac{\mathrm{RT}-a}{b}\right)^{c}} .
$$

\section{Techniques Used to Search Parameter Space}

The search for the best possible values of the free parameters of the distribution is done using the same minimization algorithm as in curve analysis. In all cases, the space is three-dimensional and all parameters are constrained to be positive or null. In the case of the Weibull, the additive parameter cannot be greater than the minimum RT.

The value that is minimized depends on the nature of the equation that needs to be fitted. For the vast majority of PDFs, the best technique to use is the maximum likelihood function. This function is known to find the best estimators when they exist and are unique. Thus, an algorithm minimizing $-L$ should tend toward a correct estimate of the free parameters if a sufficiently high number of observations are made. The likelihood function is given by the following:

$$
L(\theta)=\Pi f\left(\mathrm{RT}_{i}\right),
$$

where $\theta$ represents a given set of parameters (e.g., $\{\mu, \sigma, \tau\})$, and $\Pi$ is the product for all the observed RTs. To avoid overflow, we chose to compute a related value that has the same properties:

$$
\ln L(\theta)=\Sigma \ln \left[f\left(\mathrm{RT}_{i}\right)\right] .
$$

This statistic will be minimum for the set $\theta$ that best fits the empirical data.

If the number of observations per subject is small, it is possible to pool individual RT distributions to obtain a group distribution that preserves the shape of the component distributions. Ratcliff (1979) has shown that stable estimates of the distribution's parameters can be ob- tained using Vincent averaging (see also Dawson, 1988, and Heathcote et al., 1991).

To discover which distribution gives the best fit, a goodness-of-fit statistic called Akaike's information criterion (AIC) can be computed for each fit (reported in Maddox \& Ashby, 1993). The AIC is defined as

$$
\operatorname{AIC}\left(D_{i}\right)=-2 \ln L_{i}+2 N_{i},
$$

where $N_{i}$ is the number of free parameters in a specific distribution analysis (indexed by $i$ ) and $\ln L_{i}$ is the $\log$ likelihood of the fitted data. By including a term that penalizes a model for extra free parameters, it is possible to compare across models having a different number of parameters. The model that provides the most accurate account of the data is the one with the smallest AIC.

\section{DESCRIPTION OF PASTIS}

PASTIS (from French: Programme d'Analyse Statistique de Tendance et de dIStribution) is a UNIX-based program written in C. It can read any text file as long as data are separated by at least one space or by tab characters. Columns can be in arbitrary order, and there may be extra columns containing information not used for the analysis. Two operating modes are available: (1) In interactive mode, PASTIS displays a prompt and waits for the user to enter options, one at a time. Errors in specifying the options are more easily detected in this mode. (2) In command mode, the options are specified on the same line as the call to PASTIS and separated by at least one space. This method is faster and allows the use of PASTIS in shell files. All the following examples are issued as a command line.

Table 1 gives an overview of the type of analysis that can be performed using PASTIS. It also specifies the type of data that should be provided to it.

Following is a summary of the options necessary to make the program run:

$$
\text { -r file }
$$

: indicates which input file contains the data;

$-c x \quad: x$ indicates the total number of columns in the input file;

$-\mathrm{d} x$

$: x$ indicates which column contains the dependent variable;

-a name of analysis : name of analysis indicates the type of curve or distribution analysis (see Table 1 for the possible names; uppercase is mandatory).

Many analyses need other options. For example, pastis $-r$ data.dat -c 10 -d 3 -a PLAWCURVE $c 2$ will analyze the power law curve exhibited by the data contained in the file data.dat. This file has a total of 10 columns, and mean RTs are in column 3 (start counting at 1 ); $\mathrm{c} 2$ indicates that the session of evaluation is specified in column 2 .

Specification of the session number is mandatory for curve analysis. This specification allows the data lines in 
Table 2

Results of Curve Analyses Performed on Simulated Data

\begin{tabular}{|c|c|c|c|c|c|c|c|c|c|c|}
\hline \multirow[b]{2}{*}{ File } & \multirow{2}{*}{$\begin{array}{l}\text { Computed } \\
\text { With }\end{array}$} & \multicolumn{3}{|c|}{ Parameter Values } & \multirow{2}{*}{$\begin{array}{c}\% \\
\text { Noise } \\
\end{array}$} & \multicolumn{2}{|c|}{ Best Fit Obtained } & \multicolumn{3}{|c|}{ Parameters* } \\
\hline & & 1 & 2 & 3 & & Analysis & RMSD & 1 & 2 & 3 \\
\hline \multirow[t]{2}{*}{ PL1.dat } & Power law & 400 & 300 & 0.8 & 0 & & & 400.28 & 299.69 & 0.8015 \\
\hline & & & & & & & & & & 24 \\
\hline \multirow[t]{2}{*}{ PL2.dat } & Power law & 400 & 300 & 0.8 & 10 & /C & 14 & 459.19 & 292.83 & 2.0653 \\
\hline & & & & & & & & .35 & 12 & 0.7643 \\
\hline \multirow[t]{2}{*}{ EXPO1.dat } & Exponential & 400 & 300 & 0.4 & 0 & $\mathrm{C}$ & 4. & 328.33 & 52 & 0.5771 \\
\hline & & & & & & & & 4 & & 0.0400 \\
\hline \multirow[t]{2}{*}{ EXPO2.dat } & Exponential & 400 & 300 & 0.4 & 10 & PLAWC & 19.81 & 396.35 & 171.56 & 0.9443 \\
\hline & & & & & & EXPOC & 20.32 & 418.91 & 274.67 & 0.4744 \\
\hline
\end{tabular}

*In curve analysis, parameters 1,2 , and 3 produced by PASTIS correspond to $a, b$, and $c$, respectively.

The content of the file PLl.dat was 1700.00; 2572.30 ; 3 524.57; 4 498.96; 5 482.78; 6471.55 ; 7 463.25; $8456.84 ; 9451.73 ; 10447.55$. The semicolon denotes the new-line character. The first column is the session number, and the second is the mean RT obtained. The command line to perform the first analysis reported in Table 2 reads as follows: pastis $-r$ PL1.dat $-c 2-d 2-a$ $P L A W C$ CI

Table 2 gives the RMSD and the parameters of the curves fitted. When the file contains no noise, the fit is close to perfect, with a slight advantage for the EXPO1.dat file. Free parameters obtained reflect original parameters with less than $0.1 \%$ deviation. Results are still good when noise is added to the data. Since an average of $\pm 5 \%$ of noise was added to RTs varying from 400 to $700 \mathrm{msec}$ (with a mean near $500 \mathrm{msec}$ ), we can expect an average deviation of $25 \mathrm{msec}$. The RMSDs obtained with PASTIS are 14.22 and $20.32 \mathrm{msec}$ for the two files with noise (PL2.dat and EXPO2.dat). Clearly, data generated using a specific equation were fitted very well when using the same type of equation in analysis. However, the EXPO2.dat simulation was also well fitted by a power curve. This result calls for two important comments: First, the exponential and the power curves can be highly similar, given an appropriate set of parameters. Therefore, it is possible to obtain fits that are almost equally good for two different theoretical curves. Second, PASTIS does not provide a statistical test to discriminate among curves. This would require knowing the underlying nature of measurement noise, as discussed before.

Similar analyses were made concerning distributions. We used two files for the Weibull distribution. Both files had 34 data points, the second having $10 \%$ of uniformly distributed random noise added to the data. For the exGaussian distribution, we used the same data set as that reported in Heathcote (1996), which contained only 10 data points. The parameters used for data generation and the results of the analysis can be seen in Table 3 .

The Weibull data were better fitted when analyzed with the Weibull equation than with the ex-Gaussian equation. The same was also true when noise was added to the simulation. Application of the AIC statistic described previously indicated a difference of 35.02 in favor of the Weibull interpretation in the simulation using W1.dat $\left(\mathrm{AIC}_{\text {weibull }}=466.96\right.$ and $\left.\mathrm{AIC}_{\text {ex-gaussian }}=502.00\right)$.

The ex-Gaussian data revealed a surprise: They fit better using a Weibull equation than using the equation employed to generate the data in the first place. This result may have been due to the small number of observations in this file $(n=10)$. Since the likelihood statistic is asymptotic, it works better with a large number of observations. When analyzed using an ex-Gaussian distribution, results are exactly the same as those reported by Heathcote (1996).

\section{Availability}

PASTIS works on any workstation equipped with the cc compiler. The FORTRAN extensions must be present since STEPIT exists only in FORTRAN language. PASTIS has no limitation on the length of the input file: If the file contains fewer than 500 numbers, the data are transferred to RAM; otherwise, numbers are read directly from disk, which slows down the speed of the program (a typical analysis takes less than a minute to com-

Table 3

Results of Distribution Analyses Performed on Simulated Data

\begin{tabular}{|c|c|c|c|c|c|c|c|c|c|c|}
\hline \multirow[b]{2}{*}{ File } & \multirow{2}{*}{$\begin{array}{c}\text { Generated } \\
\text { With }\end{array}$} & \multicolumn{3}{|c|}{ Parameter Values } & \multirow{2}{*}{$\begin{array}{c}\% \\
\text { Noise }\end{array}$} & \multicolumn{2}{|c|}{ Best Fit Obtained } & \multicolumn{3}{|c|}{ Parameters* } \\
\hline & & 1 & 2 & 3 & & Analysis & -Log Likelihood & 1 & 2 & 3 \\
\hline \multirow[t]{2}{*}{ W1.dat } & Weibull & 300 & 400 & 1.3 & 0 & WEIBULL & 230.48 & 350.4 & 236.6 & 1.36 \\
\hline & & & & & & EX- & 248.00 & 518.5 & 59.9 & 154.3 \\
\hline \multirow[t]{2}{*}{ W2.dat } & Weibull & 300 & 400 & 1.3 & 10 & WEIBULL & 231.15 & 350.2 & 226.8 & 1.26 \\
\hline & & & & & & EX-GAUSS & 250.48 & 492.4 & 58.3 & 178.1 \\
\hline \multirow[t]{2}{*}{ EX-GAU.dat } & Ex-Gaussian & 500 & 50 & 100 & 0 & WEIBULL & 55.57 & 474.2 & 96.4 & 1.0 \\
\hline & & & & & & EX-GAUSS & 64.93 & 495.8 & 24.0 & 73.7 \\
\hline
\end{tabular}

*For the ex-Gaussian distribution, Parameters 1,2 , and 3 correspond to $\mu, \sigma$, and $\tau$, respectively, and for the Weibull distribution, they correspond to $a, b$, and $c$. 
plete). Input files are limited to 40 columns. The order of options does not matter except that they must precede the -a option, which specifies and triggers the analysis.

The source files, along with a compiled version for Silicon Graphics computers, come in a tar.Z file (119K). Sample files are also available in another tar. $Z$ file $(4 \mathrm{~K})$. These files can be obtained using a World-Wide Web browser at http: \\prelude.psy.umontreal.cal cousined pastis. Instructions to compile source code are also given.

\section{REFERENCES}

BATES, D. M., \& WATTS, G. W. (1988). Nonlinear regression analysis and its applications. New York: Wiley.

BRENT, R. D. (1973). Algorithms for function minimization without derivatives. Englewood Cliffs, NJ: Prentice-Hall.

CHANDLER, P. J. (1965). Subroutine STEPIT: An algorithm that finds the values of the parameters which minimize a given continuous function [Computer program]. Bloomington: Indiana University, Quantum Chemistry Program Exchange.

CONOVER, W. J. (1980). Practical nonparametric statistics. New York: Wiley.

DAwson, M. R. W. (1988). Fitting the ex-Gaussian equation to reaction time distributions. Behavior Research Methods, Instruments, \& Computers, 20, 54-57.

GEGENFURTNER, K. R. (1992). PRAXIS: Brent's algorithm for function minimization. Behavior Research Methods, Instruments, \& Computers, 24, 560-564.

HEATHCOTE, A. (1996). RTSYS: A DOS application for the analysis of reaction time data. Behavior Research Methods, Instruments, \& Computers, 28, 427-445.

Heathcote, A., \& Mewhort, D. J. K. (1995, November). The law of practice. Paper presented at the annual meeting of the Psychonomic Society, Los Angeles.

Heathcote, A., Popiel, S. J., \& Mewhort, D. J. K. (1991). Analysis of response time distributions: An example using the Stroop task. Psychological Bulletin, 109, 340-347.

HOCKLEY, W. E. (1984). Analysis of response time distributions in the study of cognitive processes. Journal of Experimental Psychology: Learning, Memory, \& Cognition, 10, 598-615.

HOHLE, R. (1965). Inferred components of reaction times as functions of foreperiod duration. Journal of Experimental Psychology, 69, 382-386.

KAIL, R., \& BISANZ, J. (1992). The information-processing perspective on cognitive development in childhood and adolescence. In R. J. Sternberg \& C. A. Berg (Eds.), Intellectual development (pp. 229-260). New York: Cambridge University Press.

Kennedy, W. J., \& Gentle, J. E. (1980). Statistical computing. New York: Marcel Dekker.

Kramer, A. F., Strayer, D. L., \& Buckley, J. (1990). Development and transfer of automatic processing. Journal of Experimental Psychology: Human Perception \& Performance, 16, 505-522.
Lassaline, M. E., \& Logan, G. D. (1993). Memory-based automaticity in the discrimination of visual numerosity. Journal of Experimental Psychology: Learning, Memory, \& Cognition, 19, 561-581.

LoGAN, G. D. (1988). Toward an instance theory of automatization. Psychological Review, 95, 492-527.

LOGAN, G. D. (1992). Shapes of reaction-time distributions and shapes of learning curves: A test of the instance theory of automaticity. Journal of Experimental Psychology: Learning, Memory, \& Cognition, 18, 883-914.

LUCE, R. D. (1986). Response times: Their role in inferring elementary mental organization. New York: Oxford University Press.

MADDOX, W. T., \& Ashry, F. G. (1993). Comparing decision bound and exemplar models of categorization. Perception \& Psychophysics, 53, 49-70.

MCGiLl, W. J. (1963). Stochastic latency mechanisms. In R. D. Luce, R. R. Bush, \& E. Galanter (Eds.), Handbook of mathematical psychology (pp. 309-360). New York: Wiley.

Newell, A., \& Rosenbloom, S. (1981). Mechanisms of skill acquisition and the law of practice. In J. R. Anderson (Ed.), Cognitive skills and their acquisition (pp. 1-56). Hillsdale, NJ: Erlbaum.

RATCLIFF, R. (1978). A theory of memory retrieval. Psychological Review, 85, 59-108.

RATCLIFF, R. (1979). Group reaction time distributions and an analysis of distribution statistics. Psychological Bulletin, 86, 446-461.

RATCLIFF, R. (1993). Methods for dealing with reaction time outliers. Psychological Bulletin, 114, 510-532.

RATCLIFF, R., \& MURDOCK, B. B. (1976). Retrieval processes in recognition memory. Psychological Review, 86, 190-214.

RESCORla, R. A., \& WAGNeR, A. R. (1973). A theory of Pavlovian conditioning: Variations in the effectiveness of reinforcement and nonreinforcement. In A. H. Black \& W. F. Prokasy (Eds.), Classical conditioning II: Current research and theory (pp. 64-99). New York: Appleton-Century-Crofts.

SternberG, S. (1969). The discovery of processing stages: Extensions of Donder's method. In W. G. Koster (Ed.), Attention and performance II (pp. 267-315). Amsterdam: North-Holland.

UlRich, R., \& Miller, J. (1994). Effects of truncation on reaction time analysis. Journal of Experimental Psychology: General, 123, 34-80.

\section{NOTES}

1. We wish to thank Gordon Logan for sending us a copy of STEPIT. 2. With a base change, Equation 5 can be rewritten as

$$
M=a+b e^{-c^{\prime} N}
$$

$a$ and $b$ are the same in both equations. The $c$ parameters are related by the formula $c^{\prime}=-\ln (1-c)$. See Kail and Bisanz (1992) for an example of the use of this formulation.

(Manuscript received April 4, 1996; revision accepted for publication July 19,1996 .) 